 \\ TATra

\section{SOME CONTINUOUS OPERATIONS ON PAIRS OF CLIQUISH FUNCTIONS}

\author{
ZBigniew Grande — Ewa StrońskA
}

\begin{abstract}
The algebraic or lattice operations in the classes of cliquish or quasicontinuous functions are well known [Z. Grande: On the maximal multiplicative family for the class of quasicontinuous functions, Real Anal. Exchange 15 (1989-1990), 437-441, Z. Grande, L. Soltysik: Some remarks on quasicontinuous real functions, Problemy Mat. 10 (1990), 79-86]. This also pertains to the symmetrical quasicontinuity or symmetrical cliquishness [Z. Grande: On the maximal additive and multiplicative families for the quasicontinuities of Piotrowski and Vallin, Real Anal. Exchange 32 (2007), 511-518]. In this article, we examine the superpositions $F(f, g)$, where $F$ is a continuous operation and $f, g$ are cliquish (symmetrically cliquish) or $f$ is continuous ( $f$ is symmetrically quasicontinuous with continuous sections) and $g$ is quasicontinuous (symmetrically quasicontinuous).
\end{abstract}

Let $\left(X, T_{X}\right)$ and $\left(Y, T_{Y}\right)$ be topological spaces, let $(Z, \rho)$ and $\left(Z_{i}, \rho_{i}\right),(i=1,2)$ be metric spaces.

Definition ([10, 11]). A function $f: X \rightarrow Z$ is said to be

- quasicontinuous (cliquish) at a point $x \in X$ if for every set $U \in T_{X}$ containing $x$ and for each positive real $\varepsilon$ there is a nonempty set $U^{\prime} \in T_{X}$ contained in $U$ such that $f\left(U^{\prime}\right) \subset K(f(x), \varepsilon)=\{t \in Z ; \rho(t, f(x))<\varepsilon\}\left(\operatorname{diam}\left(f\left(U^{\prime}\right)\right)=\right.$ $\left.\sup \left\{\rho(f(t), f(u)) ; t, u \in U^{\prime}\right\}<\varepsilon\right)$.

Definition. A function $f: X \times Y \rightarrow Z$ is said to be:

- quasicontinuous at $(x, y)$ with respect to $x$ (alternatively $y$ ) if for every set $U \times V \in T_{X} \times T_{Y}$ containing $(x, y)$ and for each positive real $\varepsilon$ there are nonempty sets $U^{\prime} \in T_{X}$ contained in $U$ and $V^{\prime} \in T_{Y}$ contained in $V$ such that $x \in U^{\prime}$ (alternatively $\left.y \in V^{\prime}\right)$ and $f\left(U^{\prime} \times V^{\prime}\right) \subset K(f(x, y), \varepsilon)([10]-[12])$;

2000 Mathematics Subject Classification: 26A15, 26A21, 54C05.

Keywords: quasicontinuity, symmetrical quasicontinuity, cliquishness, symmetrical cliquishness, strong quasicontinuity, strong cliquishness, continuous operations, maximal family for operation. 


\section{ZBIGNIEW GRANDE — EWA STROŃSKA}

- cliquish at $(x, y)$ with respect to $x$ (alternatively $y$ ) if for every set $U \times V \in T_{X} \times T_{Y}$ containing $(x, y)$ and for each positive real $\varepsilon$ there are nonempty sets $U^{\prime} \in T_{X}$ contained in $U$ and $V^{\prime} \in T_{Y}$ contained in $V$ such that $x \in U^{\prime}$ (alternatively $\left.y \in V^{\prime}\right)$ and $\left.\operatorname{diam}\left(f\left(U^{\prime} \times V^{\prime}\right)\right)<\varepsilon\right)([3])$;

- symmetrically quasicontinuous (symmetrically cliquish) at $(x, y)$ if it is quasicontinuous (cliquish) at $(x, y)$ with respect to $x$ and with respect to $y$.

Evidently the cliquishness (the quasicontinuity) with respect to $x$ (or to $y$ ) of a function $f: X \times Y \rightarrow Z$ implies its cliquishness (its quasicontinuity) and the respective quasicontinuities imply the respective cliquishness.

\section{The superposition $x \rightarrow F(f(x), g(x))$ on pairs of cliquish functions $(f, g)$}

We start from the well-known examples. Let $\left(a_{n}\right)$ be an enumeration of all rationals such that $a_{n} \neq a_{m}$ for $n \neq m$.

1. Let $f_{1}\left(a_{n}\right)=\frac{1}{n}$ for $n \geq 1$. Then $f_{1}: \mathbb{Q} \rightarrow(0, \infty)$ is a cliquish function (we consider the spaces $\mathbb{Q}$ and $(0, \infty)$ with the natural metric), but its inversion

$$
\frac{1}{f_{1}\left(a_{n}\right)}=n \quad \text { for } \quad n \geq 1
$$

is not cliquish at any point.

2. Observe that the given $F_{1}:(0, \infty)^{2} \rightarrow(0, \infty)$ defined by the formula

$$
F_{1}(u, v)=\frac{1}{u+v}
$$

is continuous, but the superposition

is not cliquish.

$$
F_{1}\left(f_{1}\left(a_{n}\right), f_{1}\left(a_{n}\right)\right)=\frac{n}{2}, \quad n \geq 1,
$$

3. Analogously, the given $g_{1}: \mathbb{Q}^{2} \rightarrow(0, \infty)$ defined by the formula

$$
g_{1}\left(a_{n}, a_{m}\right)=\frac{1}{n m} \quad \text { for } n, m \geq 1,
$$

is symmetrically cliquish, but the superposition

$$
F_{1}\left(g_{1}, g_{1}\right)=\frac{1}{2 g_{1}}
$$

is not cliquish at any point. 
So, there are cliquish functions $f, g: \mathbb{Q} \rightarrow(0, \infty)$ (symmetrically cliquish functions $\left.f, g: \mathbb{Q}^{2} \rightarrow(0,1)\right)$ and a continuous function $F:(0, \infty)^{2} \rightarrow(0, \infty)$ such that the superposition $F(f, g)$ is not cliquish. Moreover the following theorems are true.

TheOREM 1. Let $F: Z_{1} \times Z_{2} \rightarrow Z$ be a uniformly continuous function. If the functions $f: X \rightarrow Z_{1}$ and $g: X \rightarrow Z_{2}$ are cliquish at each point $x \in X$ then the function $h(x)=F(f(x), g(x))$ for $x \in X$ is also cliquish at each point $x$.

Proof. Fix a real $\varepsilon>0$, a point $x \in X$ and an open set $U \in T_{X}$ with $x \in U$. From the uniform continuity of $F$ there is a real $\delta>0$ such that for $z_{1}, z_{2} \in Z_{1}$ and $z_{3}, z_{4} \in Z_{2}$ if $\rho_{1}\left(z_{1}, z_{2}\right)<\delta$ and $\rho_{2}\left(z_{3}, z_{4}\right)<\delta$ then $\rho\left(F\left(z_{1}, z_{3}\right), F\left(z_{2}, z_{4}\right)\right)<\frac{\varepsilon}{2}$. Since $f$ is cliquish at $x$, there is a nonempty open set $U^{\prime} \in T_{X}$ such that

$$
U^{\prime} \subset U \text { and } \operatorname{diam}\left(\mathrm{f}\left(\mathrm{U}^{\prime}\right)\right)<\delta .
$$

Fix a point $v \in U^{\prime}$. Since $g$ is cliquish at $v$, there is a nonempty open set $U^{\prime \prime} \in T_{X}$ such that

$$
U^{\prime \prime} \subset U^{\prime} \text { and } \operatorname{diam}\left(\mathrm{g}\left(\mathrm{U}^{\prime \prime}\right)\right)<\delta .
$$

Observe that for $x_{1}, x_{2}$ belonging to $U^{\prime \prime}$ we have

$$
\rho_{1}\left(f\left(x_{1}\right), f\left(x_{2}\right)\right)<\delta \quad \text { and } \quad \rho_{2}\left(g\left(x_{1}\right), g\left(x_{2}\right)\right)<\delta,
$$

thus the inequality

$$
\rho\left(F\left(f\left(x_{1}\right), g\left(x_{1}\right), F\left(f\left(x_{2}\right), g\left(x_{2}\right)\right)\right)<\frac{\varepsilon}{2}\right.
$$

is true. So, $\operatorname{diam}\left(h\left(U^{\prime \prime}\right)\right) \leq \frac{\varepsilon}{2}<\varepsilon$ and the proof is finished.

Theorem 2. Let $F: Z_{1} \times Z_{2} \rightarrow Z$ be a uniformly continuous function. If the functions $f: X \times Y \rightarrow Z_{1}$ and $g: X \times Y \rightarrow Z_{2}$ are cliquish at each point $(x, y) \in X \times Y$ with respect to $x$ (alternatively to $y)$, then the function $h(x, y)=$ $F(f(x, y), g(x, y))$ for $(x, y) \in X \times Y$ is also cliquish at each point $(x, y)$ with respect to $x$ (alternatively to $y$ ).

Proof. Fix a real $\varepsilon>0$, a point $(x, y) \in X \times Y$ and open sets $U \in T_{X}$ and $V \in T_{Y}$ with $(x, y) \in U \times Y$. From the uniform continuity of $F$ there is a real $\delta>0$ such that for $z_{1}, z_{2} \in Z_{1}$ and $z_{3}, z_{4} \in Z_{2}$ if

$$
\rho_{1}\left(z_{1}, z_{2}\right)<\delta \quad \text { and } \quad \rho_{2}\left(z_{3}, z_{4}\right)<\delta \quad \text { then } \rho\left(F\left(z_{1}, z_{3}\right), F\left(z_{2}, z_{4}\right)\right)<\frac{\varepsilon}{2} \text {. }
$$

Since $f$ is cliquish at $(x, y)$ with respect to $x$, there are nonempty open sets $U^{\prime} \in T_{X}$ and $V^{\prime} \in T_{Y}$ such that

$$
x \in U^{\prime} \subset U, V^{\prime} \subset V \text { and } \operatorname{diam}\left(\mathrm{f}\left(\mathrm{U}^{\prime} \times \mathrm{V}^{\prime}\right)\right)<\delta .
$$




\section{ZBIGNIEW GRANDE — EWA STROŃSKA}

Fix a point $v \in V^{\prime}$. Since $g$ is cliquish at $(x, v)$ with respect to $x$, there are nonempty open sets $U^{\prime \prime} \in T_{X}$ and $V^{\prime \prime} \in T_{Y}$ such that

$$
x \in U^{\prime \prime} \subset U^{\prime}, V^{\prime \prime} \subset V^{\prime} \text { and } \operatorname{diam}\left(\mathrm{g}\left(\mathrm{U}^{\prime \prime} \times \mathrm{V}^{\prime \prime}\right)\right)<\delta .
$$

Observe that for $\left(x_{1}, y_{1}\right)$ and $\left(x_{2}, y_{2}\right)$ belonging to $U^{\prime \prime} \times V^{\prime \prime}$ we have

$$
\rho_{1}\left(f\left(x_{1}, y_{1}\right), f\left(x_{2}, y_{2}\right)\right)<\delta \quad \text { and } \quad \rho_{2}\left(g\left(x_{1}, y_{1}\right), g\left(x_{2}, y_{2}\right)\right)<\delta,
$$

thus the inequality

$$
\rho\left(F\left(f\left(x_{1}, y_{1}\right), g\left(x_{1}, y_{1}\right)\right), F\left(f\left(x_{2}, y_{2}\right), g\left(x_{2}, y_{2}\right)\right)\right)<\frac{\varepsilon}{2}
$$

is true. So, $\operatorname{diam}\left(h\left(U^{\prime \prime} \times V^{\prime \prime}\right)\right) \leq \frac{\varepsilon}{2}<\varepsilon$ and in the case where $f$ and $g$ are cliquish at $(x, y)$ with respect to $x$ the proof is finished. In the alternative case where $f$ and $g$ are cliquish at $(x, y)$ with respect to $y$ the proof is analogous.

\section{Corollaries and remarks}

From Theorem 1 we obtain

Corollary 1. Let $F: Z_{1} \times Z_{1} \rightarrow Z$ be a continuous function. If the functions $f, g: X \times Y \rightarrow Z_{1}$ are cliquish at each point $(x, y) \in X \times Y$ with respect to $x$ (alternatively to $y)$ and if the closures $\mathrm{cl}(\mathrm{f}(\mathrm{X} \times \mathrm{Y}))$ and $\mathrm{cl}(\mathrm{g}(\mathrm{X} \times \mathrm{Y}))$ are compact, then the functions $h_{1}(x, y)=F(f(x, y), g(x, y))$ and $h_{2}(x, y)=F(g(x, y), f(x, y))$ for $(x, y) \in X \times Y$ are also cliquish at each point $(x, y)$ with respect to $x$ (alternatively to $y)$.

From Theorem 2 we have

Corollary 2. Let $F: Z_{1} \times Z_{1} \rightarrow Z$ be a uniformly continuous function. If the functions $f, g: X \times Y \rightarrow Z_{1}$ are symmetrically cliquish at each point $(x, y) \in X \times Y$, then the functions $h_{1}(x, y)=F(f(x, y), g(x, y))$ and $h_{2}(x, y)=$ $F(g(x, y), f(x, y))$ for $(x, y) \in X \times Y$ are also symmetrically cliquish at each point $(x, y)$.

In the case $Z=Z_{1}=\mathbb{R}$ and $\rho\left(z_{1}, z_{2}\right)=\rho_{1}\left(z_{1}, z_{2}\right)=\left|z_{1}-z_{2}\right|$, the functions $F_{1}\left(z_{1}, z_{2}\right)=\max \left(z_{1}, z_{2}\right)$ and $F_{2}\left(z_{1}, z_{2}\right)=\min \left(z_{1}, z_{2}\right)$ are uniformly continuous. So, from Theorem 2 we obtain

Corollary 3. If the functions $f, g: X \times Y \rightarrow \mathbb{R}$ are cliquish at each point $(x, y) \in X \times Y$ with respect to $x$ (alternatively to $y$ ), then the functions $\max (f, g)$ and $\min (f, g)$ are also cliquish at each point $(x, y)$ with respect to $x$ (alternatively with respect to $y)$.

In the case where $(Z,\|\cdot\|)=\left(Z_{1},\|\cdot\|\right)$ is a normed space, for all reals $r_{1}, r_{2}$ the function $F\left(z_{1}, z_{2}\right)=r_{1} z_{1}+r_{2} z_{2}$ is uniformly continuous. So, from Theorem 2 , we obtain 
Corollary 4. If $(Z,\|\cdot\|)$ is a normed space and the functions $f, g: X \times Y \rightarrow Z$ are cliquish at each point $(x, y) \in X \times Y$ with respect to $x$ (alternatively with respect to $y$ ), then for all real $r_{1}, r_{2}$ the function $r_{1} f+r_{2} g$ is also cliquish at each point $(x, y) \in X \times Y$ with respect to $x$ (alternatively with respect to $y$ ).

From Corollary 1 for the case $Z=Z_{1}=\mathbb{R}$ and $\rho\left(z_{1}, z_{2}\right)=\rho_{1}\left(z_{1}, z_{2}\right)=\left|z_{1}-z_{2}\right|$ it follows the following

COROLlARY 5. If the bounded functions $f, g: X \times Y \rightarrow \mathbb{R}$ are cliquish at each point $(x, y) \in X \times Y$ with respect to $x$ (alternatively with respect to $y$ ), then the product $f g$ is also cliquish at each point $(x, y)$ with respect to $x$ (alternatively with respect to $y$ ).

The product $F(x, y)=x y$ for $x, y \in \mathbb{R}$ is not uniformly continuous in $\mathbb{R}^{2}$ with the natural metric. Similarly, the scalar product $F(x, y)=(x \mid y)$ for $x, y \in \mathbb{R}^{n}$ is not uniformly continuous in $\mathbb{R}^{2 n}$ with natural metric. So, for the proof of the cliquishness (or of the symmetrical cliquishness) of the product or the scalar product of two cliquish (or symmetrically cliquish) functions Theorem 2 is not sufficient, however, we can use the following remarks.

Remark 1. Suppose that $\left(X, T_{X}\right)$ is a Baire space. If the functions $f, g: X \rightarrow Z_{1}$ are cliquish and $F: Z_{1} \times Z_{1} \rightarrow Z$ is a continuous function then the superposition $h(x)=F(f(x), g(x))$ for $x \in X$ is cliquish.

P r o of. Denote by $C(f)(C(g))$ the set of all continuity points of $f$ (of $g$ ). Since $\left(X, T_{X}\right)$ is a Baire space, the sets $C(f)$ and $C(g)$ are residual in $X$ (see [2], [1] ). Consequently, the intersection $C(f) \cap C(g)$ is residual in $X$ and $C(f) \cap C(g) \subset$ $C(F(f, g))$, so the superposition $F(f, g)$ is cliquish.

Remark 2. Suppose $\left(X, T_{X}\right)$ (alternatively $\left.\left(Y, T_{Y}\right)\right)$ is a Baire space and functions $f, g: X \times Y \rightarrow Z_{1}$ are cliquish with respect to $y$ (alternatively to $x$ ). If a function $F: Z_{1} \times Z_{1} \rightarrow Z$ is continuous then the superpositions $h_{1}(x)=$ $F(f(x, y), g(x, y))$ and $h_{2}(x)=F(g(x, y), f(x, y))$ for $x \in X$ are cliquish with respect to $y$ (alternatively to $x$ ).

Pr o of. Similarly as in the proof of the previous remark, we observe that the sections $(C(f))^{y}$ and $(C(g))^{y}, y \in Y$, are residual in $X$ (see [6]). Consequently, the sections $(C(F(f, g)))^{y} \supset(C(f) \cap C(g))^{y}, y \in Y$, are residual in $X$ and the superposition $h_{1}$ is cliquish with respect to $y$. Similarly, we can prove the cliquishness of $h_{1}$ with respect to $x$ and the cliquishness of $h_{2}$ with respect to $x$ and to $y$.

Corollary 6. Suppose $\left(X, T_{X}\right)$ and $\left(Y, T_{Y}\right)$ are Baire spaces and the functions $f, g: X \times Y \rightarrow Z_{1}$ are symmetrically cliquish. If a function $F: Z_{1} \times Z_{1} \rightarrow Z$ $i$ continuous then the superposition $h(x)=F(f(x, y), g(x, y))$ is symmetrically cliquish. 


\section{ZBIGNIEW GRANDE — EWA STROŃSKA}

\section{The superposition $F(f, g)$, where one of the functions $f$ and $g$ is quasicontinuous and the second is continuous}

Recall that there are quasicontinuous real-valued functions whose sum is not quasicontinuous (see [8]). We start from the following easy observation:

Remark 3. Let $F: Z_{1} \times Z_{1} \rightarrow Z$ be a continuous function. If a function $f: X \rightarrow Z_{1}$ is quasicontinuous at a point $x_{0} \in X$ and a function $g: X \rightarrow Z_{1}$ is continuous at $x_{0}$ then the functions $h_{1}(x)=F(f(x), g(x))$ and $h_{2}(x)=$ $F(g(x), f(x)), x \in X$, are also quasicontinuous at the point $x_{0}$.

P r o of. Both functions $k_{1}(x)=(f(x), g(x))$ and $k_{2}(x)=(g(x), f(x)), x \in X$, are quasicontinuous at $x_{0}$, so their superpositions $F\left(k_{1}\right)$ and $F\left(k_{2}\right)$ with a continuous function $F$ are quasicontinuous at $x_{0}$.

Theorem 3. Let $F: Z_{1} \times Z_{1} \rightarrow Z$ be a continuous function. If the functions $f, g: X \times Y \rightarrow Z_{1}$ are quasicontinuous at each point $(x, y) \in X \times Y$ with respect to $x$ (alternatively with respect to $y$ ) and at each point $(x, y) \in X \times Y$ at least one of them has continuous vertical section (horizontal section) then the functions $h_{1}(x, y)=F(f(x, y), g(x, y))$ and $h_{2}(x, y)=F(g(x, y), f(x, y))$ for $(x, y) \in$ $X \times Y$ are also quasicontinuous at each point $(x, y)$ with respect to $x$ (alternatively with respect to $y$ ).

Proof. Fix a real $\varepsilon>0$, a point $(x, y) \in X \times Y$ and open sets $U \in T_{X}$ and $V \in T_{Y}$ with $(x, y) \in U \times V$. We can assume that the vertical section $g_{x}$ is continuous at $y$. From the continuity of $F$ at the point $(f(x, y), g(x, y))$, there is a real $\delta>0$ such that for $z_{1}, z_{2} \in Z_{1}$ if $\rho_{1}\left(z_{1}, f(x, y)\right)<\delta$ and $\rho_{1}\left(z_{2}, g(x, y)\right)<\delta$, then

$$
\rho\left(F\left(z_{1}, z_{2}\right), F(f(x, y), g(x, y))\right)<\frac{\varepsilon}{3} .
$$

From the continuity of the section $g_{x}$ at $y$, it follows that there is a set $V_{1} \in T_{Y}$ containing $y$ such that $V_{1} \subset V$ and $\rho_{1}(g(x, w), g(x, y))<\delta$ for $w \in V_{1}$. Since $f$ is quasicontinuous at $(x, y)$ with respect to $x$, there are nonempty open sets $U^{\prime} \in T_{X}$ and $V^{\prime} \in T_{Y}$ such that

$$
x \in U^{\prime} \subset U, V^{\prime} \subset V_{1} \text { and } f\left(U^{\prime} \times V^{\prime}\right) \subset K(f(x, y), \delta) .
$$

Fix a point $v \in V^{\prime}$. By the continuity of $F$ at $(f(x, v), g(x, v))$, there is a real $\delta_{1} \in$ $(0, \delta)$ such that for $z_{3}, z_{4} \in Z_{1}$ if $\rho_{1}\left(z_{3}, f(x, v)\right)<\delta_{1}$ and $\rho_{1}\left(z_{4}, g(x, v)\right)<\delta_{1}$, then

$$
\rho\left(F\left(z_{3}, z_{4}\right), F(f(x, v), g(x, v))\right)<\frac{\varepsilon}{3} .
$$

Since $g$ is quasicontinuous at $(x, v)$ with respect $x$, there are open sets $U^{\prime \prime} \in T_{X}$ and $V^{\prime \prime} \in T_{Y}$ such that

$$
\left.x \in U^{\prime \prime} \subset U^{\prime}, V^{\prime \prime} \subset V^{\prime} \text { and } g\left(U^{\prime \prime} \times V^{\prime \prime}\right)\right) \subset K\left(g(x, v), \delta_{1}\right) .
$$


Observe that for $\left(x_{1}, y_{1}\right)$ belonging to $U^{\prime \prime} \times V^{\prime \prime}$ we have

$$
\rho_{1}\left(f\left(x_{1}, y_{1}\right), f(x, y)\right)<\delta \quad \text { and } \quad \rho_{1}\left(g\left(x_{1}, y_{1}\right), g(x, v)\right)<\delta_{1},
$$

thus the inequalities

$$
\begin{aligned}
& \rho\left(F\left(f\left(x_{1}, y_{1}\right), g\left(x_{1}, y_{1}\right)\right), F(f(x, y), g(x, y))\right) \\
\leq & \rho\left(F\left(f\left(x_{1}, y_{1}\right), g\left(x_{1}, y_{1}\right)\right), F(f(x, v), g(x, v))\right) \\
& +\rho(F(f(x, v), g(x, v)), F(f(x, y), g(x, y))) \\
< & \frac{\varepsilon}{3}+\frac{\varepsilon}{3}=\frac{2 \varepsilon}{3}<\varepsilon
\end{aligned}
$$

are true. So, $h_{1}\left(U^{\prime \prime} \times V^{\prime \prime}\right) \subset K\left(h_{1}(x, y), \varepsilon\right)$, and the proof for $h_{1}$ is completed. Proofs of the quasicontinuity of $h_{2}$ at each point $(x, y)$ with respect to $x$ and of the quasicontinuity with respect to $y$ are analogous.

Corollary 7. Let $F: Z_{1} \times Z_{1} \rightarrow Z$ be a continuous function. If the functions $f, g: X \times Y \rightarrow Z_{1}$ are symmetrically quasicontinuous at each point $(x, y) \in$ $X \times Y$ and the sections $g_{x}$ and $g^{y}$ are continuous, then the function $h(x, y)=$ $F(f(x, y), g(x, y))$ for $(x, y) \in X \times Y$ is also symmetrically quasicontinuous at each point $(x, y)$.

In the case $Z=Z_{1}=\mathbb{R}$ and $\rho\left(z_{1}, z_{2}\right)=\rho_{1}\left(z_{1}, z_{2}\right)=\left|z_{1}-z_{2}\right|$, the functions $F_{1}\left(z_{1}, z_{2}\right)=\max \left(z_{1}, z_{2}\right)$ and $F_{2}\left(z_{1}, z_{2}\right)=\min \left(z_{1}, z_{2}\right)$ are uniformly continuous. So, from Theorem 3 , we obtain

CoRollary 8. If the functions $f, g: X \times Y \rightarrow \mathbb{R}$ are quasicontinuous at each point $(x, y) \in X \times Y$ with respect to $x$ (alternatively to $y$ ) and if the function $g: X \times Y \rightarrow \mathbb{R}$ has continuous vertical (horizontal) sections then the functions $\max (f, g), \min (f, g)$ and $f g$ are also quasicontinuous at each point $(x, y)$ with respect to $x$ (alternatively with respect to $y$ ).

In the case where $(Z,\|\cdot\|)=\left(Z_{1},\|\cdot\|\right)$ is a normed space then for all reals $r_{1}, r_{2}$ the function $F\left(z_{1}, z_{2}\right)=r_{1} z_{1}+r_{2} z_{2}$ is uniformly continuous. So, from Theorem 3, we obtain

Corollary 9. If $(Z,\|\cdot\|)$ is a normed space, the functions $f, g: X \times Y \rightarrow Z$ are quasicontinuous at each point $(x, y) \in X \times Y$ with respect to $x$ (alternatively with respect to $y$ ) and the function $f: X \times Y \rightarrow Z$ has continuous vertical (horizontal) sections, then for all real $r_{1}, r_{2}$, the function $r_{1} f+r_{2} g$ is also quasicontinuous at each point $(x, y) \in X \times Y$ with respect to $x$ (alternatively with respect to $y$ ). 


\section{ZBIGNIEW GRANDE — EWA STROŃSKA}

\section{Continuous operations and kinds of the cliquishness and quasicontinuity}

Consider the real line $\mathbb{R}$ with the natural metric and denote by $T_{d}$ the density topology in $\mathbb{R}([1],[13])$. A special kind of the cliquishness (the quasicontinuity) of the functions from $\mathbb{R}$ to $\mathbb{R}$ is a strong cliquishness (strong quasicontinuity).

Definition ([7]). A function $f: \mathbb{R} \rightarrow \mathbb{R}$ is said to be strongly quasicontinuous (resp. strongly cliquish) at a point $x$ if for each positive real $\eta$ and for each set $A \in T_{d}$ containing $x$, there is an open interval $I$ such that $I \cap A \neq \emptyset$ and $f(A \cap I) \subset(f(x)-\eta, f(x)+\eta)(\operatorname{diam}(f(A \cap I))<\eta)$.

TheOREM 4. Let $F: \mathbb{R}^{2} \rightarrow \mathbb{R}$ be a uniformly continuous function. If the functions $f, g: \mathbb{R} \rightarrow \mathbb{R}$ are strongly cliquish at each point $x \in \mathbb{R}$, then the function $h(x)=F(f(x), g(x))$ for $x \in \mathbb{R}$ is also strongly cliquish at each point $x$.

Pr o of. Fix a real $\varepsilon>0$, a point $x \in X$ and a set $U \in T_{d}$ with $x \in U$. From the uniform continuity of $F$ there is a real $\delta>0$ such that for $z_{1}, z_{2}, z_{3}, z_{4} \in \mathbb{R}$ if

$$
\max \left(\left|z_{1}-z_{2}\right|,\left|z_{3}-z_{4}\right|\right)<\delta, \quad \text { then }\left|F\left(z_{1}, z_{3}\right)-F\left(z_{2}, z_{4}\right)\right|<\frac{\varepsilon}{2} .
$$

Since $f$ is strongly cliquish at $x$, there is an open interval $I$ such that

$$
I \cap U \neq \emptyset \text { and } \operatorname{diam}(\mathrm{f}(\mathrm{I} \cap \mathrm{U}))<\delta .
$$

Fix a point $v \in U \cap I$. Since $g$ is strongly cliquish at $v$, there is an open interval $I^{\prime} \subset I$ such that

$$
I^{\prime} \cap U \neq \emptyset \text { and } \operatorname{diam}\left(\mathrm{g}\left(\mathrm{U} \cap \mathrm{I}^{\prime}\right)\right)<\delta .
$$

Observe that for any points $x_{1}, x_{2}$ belonging to $U \cap I^{\prime}$, we have

$$
\mid f\left(x_{2}\right)-f\left(x_{1} \mid<\delta \text { and }\left|g\left(x_{1}\right)-g\left(x_{2}\right)\right|<\delta,\right.
$$

thus the inequality

$$
\left|F\left(f\left(x_{1}\right), g\left(x_{1}\right)\right)-F\left(f\left(x_{2}\right), g\left(x_{2}\right)\right)\right|<\frac{\varepsilon}{2}
$$

is true. So, $\operatorname{diam}\left(h\left(U^{\prime} \cap I\right)\right) \leq \frac{\varepsilon}{2}<\varepsilon$. Thus, the function $h$ is strongly cliquish and the proof is finished.

TheOREM 5. Let $F: \mathbb{R}^{2} \rightarrow \mathbb{R}$ be a continuous function, let $f: \mathbb{R} \rightarrow \mathbb{R}$ be a strongly quasicontinuous function and let $g: \mathbb{R} \rightarrow \mathbb{R}$ be a continuous function. Then the functions $h_{1}(x)=F(f(x), g(x))$ and $h_{2}(x)=F(g(x), f(x))$ are strongly quasicontinuous. 
Proof. Fix a point $x \in \mathbb{R}$, a set $A \in T_{d}$ containing $x$ and a positive real $\varepsilon$. From the continuity of $F$, there is a real $\delta>0$ such that for $u, v \in \mathbb{R}$ if

$$
\max (|u-f(x)|,|v-g(x)|)<\delta, \quad \text { then }|F(u, v)-F(f(x), g(x))|<\varepsilon .
$$

Since $g$ is continuous there is an open interval

$$
I \ni x \quad \text { with } \quad g(I) \subset(g(x)-\delta, g(x)+\delta) .
$$

From the strong quasicontinuity of $f$, it follows that there is an open interval $I_{1} \subset I$ such that

$$
I_{1} \cap A \neq \emptyset \quad \text { and } \quad f\left(I_{1} \cap A\right) \subset(f(x)-\delta, f(x)+\delta) .
$$

Consequently, for $y \in I_{1} \cap A$ we have

$|f(y)-f(x)|<\delta,|g(y)-g(x)|<\delta \quad$ and $|F(f(y), g(y))-F(f(x), g(x))|<\varepsilon$.

Thus, $h_{1}$ (and similarly $h_{2}$ ) is strongly quasicontinuous.

The paper pertains to continuous operations on pairs of functions. However, answering to a question of the referee, we show that quasicontinuity of $F$ in Theorem 3, 4 and 5 and Remark 3 is not sufficient.

Indeed, the function

$$
F(u, v)=\left\{\begin{array}{ccl}
0 & \text { if } & v=u \text { and } u \in \mathbb{Q}, \\
0 & \text { if } & v<u, \\
1, & \text { othervise on } & \mathbb{R}^{2}
\end{array}\right.
$$

is quasicontinuous and the superposition $(x, y) \rightarrow F(x, x)$ is not cliquish.

\section{Maximal F-families}

Analogously as in the cases of maximal families for the addition and the multiplication of functions, we can define maximal $F$-families. Limit our consideration to continuous operations $F: \mathbb{R}^{2} \rightarrow \mathbb{R}$. If $\Phi$ is a family of functions from $\mathbb{R}$ to $\mathbb{R}$, then put

$$
\begin{aligned}
& \operatorname{Max}_{\mathrm{lF}}(\Phi)=\{f \in \Phi ; F(f, g) \in \Phi \quad \text { for each } g \in \Phi\} \text {, } \\
& \operatorname{Max}_{\mathrm{rF}}(\Phi)=\{f \in \Phi ; F(g, f) \in \Phi \quad \text { for each } g \in \Phi\} \text {. }
\end{aligned}
$$

Observe that there are families $\Phi$ for which $\operatorname{Max}_{1 \Phi} \neq \operatorname{Max} \operatorname{Mar}_{\mathrm{r}}$. For example,

1. If $F_{3}(u, v)=u^{2}$ and $\Phi=b \Delta^{\prime}$ is the family of all bounded derivatives then $\operatorname{Max}_{\mathrm{lF}_{3}}\left(\mathrm{~b} \Delta^{\prime}\right)$ is the family of all bounded approximately continuous functions $\left(\left[1\right.\right.$, , 9]) and $\operatorname{Max}_{\mathrm{rF}_{3}}\left(\mathrm{~b} \Delta^{\prime}\right)=\emptyset$. 


\section{ZBIGNIEW GRANDE — EWA STROŃSKA}

2. Let $\mathcal{Q}$ denotes the family of all quasicontinuous functions from $\mathbb{R}$ to $\mathbb{R}$. Observe that for the operation $F_{4}(u, v)=u+v^{2}$ and the family $\mathcal{Q}$ we have also $\operatorname{Max}_{\mathrm{lF}_{4}}(\mathcal{Q}) \neq \operatorname{Max}_{\mathrm{rF}_{4}}(\mathcal{Q})$. Indeed, let

$$
f(x)=1 \quad \text { for } x \geq 0 \quad \text { and } \quad f(x)=-1 \quad \text { for } x<0 .
$$

Since $f^{2}$ is constant and for the quasicontinuous function

$$
g(x)=2 \text { for } x>0 \quad \text { and } \quad g(x)=1 \text { for } x \leq 0,
$$

the sum $f+g^{2} \notin \mathcal{Q}$, the function $f \in \operatorname{Max}_{\mathrm{rF}_{4}}(\mathcal{Q}) \backslash \operatorname{Max}_{\mathrm{lF}_{4}}(\mathcal{Q})$.

Let $\mathcal{C}$ denote the family of all continuous functions from $\mathbb{R}$ to $\mathbb{R}$. From Remark 3 it follows that for each continuous operation $F: \mathbb{R}^{2} \rightarrow \mathbb{R}$ we have

$$
\mathcal{C} \subset \operatorname{Max}_{\mathrm{lF}}(\mathcal{Q}) \cap \operatorname{Max}_{\mathrm{rF}}(\mathcal{Q}) .
$$

Remark 4. Let $F(u, v)=a u+b v$, where $a, b \neq 0$. Then

$$
\operatorname{Max}_{\mathrm{lF}}(\mathcal{Q})=\operatorname{Max}_{\mathrm{rF}}(\mathcal{Q})=\mathcal{C}
$$

Proof. It is sufficient to prove $\operatorname{Max}_{\mathrm{lF}}(\mathcal{Q})=\operatorname{Max}_{\mathrm{rF}}(\mathcal{Q}) \subset \mathcal{C}$. Fix a function $f \in \mathcal{Q} \backslash \mathcal{C}$. If there is a point $x \in \mathbb{R}$ at which $f$ has at least one limit number $y_{1} \in \mathbb{R}$ different from $f(x)$, then we put

$$
g(t)=\frac{-a y_{1}}{b} \quad \text { for } t=x \quad \text { and } \quad g(t)=\frac{-a f(t)}{b} \text { for } t \neq x .
$$

Then $g \in \mathcal{Q}, F(f(t), g(t))=a f(t)-a f(t)=0$ for $t \neq x$ and $F(f(x), g(x))=$ $a f(x)-a y_{1} \neq 0$. So, the superposition $t \rightarrow F(f(t), g(t)), t \in \mathbb{R}$, is not quasicontinuous.

In the opposite case there are a point $x$ and a sequence of points $x_{n} \neq x$ such that $\lim _{n \rightarrow \infty} x_{n}=x$ and $\lim _{n \rightarrow \infty}\left|f\left(x_{n}\right)\right|=\infty$. Since $f$ is quasicontinuous at the points $x_{n}, n \geq 1$, there are pairwise disjoint open intervals $I_{n}=\left(a_{n}, b_{n}\right)$ such that $\lim _{n \rightarrow \infty} a_{n}=\lim _{n \rightarrow \infty} b_{n}=x, \lim _{n \rightarrow \infty}\left|f\left(a_{n}\right)\right|=\infty$ and $\operatorname{diam}\left(f\left(I_{n}\right)\right)<\frac{1}{n}$ for $n \geq 1$. For each integer $n \geq 1$ we find a closed interval $J_{n} \subset I_{n}$. Let $c \in \mathbb{R}$ be a real such that $a f(x)-b c \neq 0$. Put

$$
g(t)=-c \quad \text { for } \quad t \in J_{n}, n \geq 1, \quad g(x)=-c,
$$

and

$$
g(t)=\frac{-a f(t)}{b}, \quad \text { otherwise on } \mathbb{R} .
$$

Then $g \in \mathcal{Q}$ and the superposition $t \rightarrow F(f(t), g(t))$ is not quasicontinuous at the point $x$. This proves that $\operatorname{Max}_{\mathrm{lF}}(\mathcal{Q})=\mathcal{C}$. The proof of the equality $\operatorname{Max}_{\mathrm{rF}}(\mathcal{Q})=\mathcal{C}$ is similar.

In [5], it is proved that for the operation $F(u, v)=u v$ we have

$$
\operatorname{Max}_{\mathrm{lF}}(\mathcal{Q})=\operatorname{Max}_{\mathrm{rF}}(\mathcal{Q}) \neq \mathcal{C} .
$$




\section{SOME CONTINUOUS OPERATIONS}

\section{REFERENCES}

[1] BRUCKNER, A.: Differentiation of Real Functions. Lectures Notes in Math., Vol. 659, Springer-Verlag, Berlin, 1978.

[2] EWERT, J.-LIPIŃSKI, J.: On points of continuity, quasicontinuity and cliqiushnees of real functions, Real Anal. Exchange 8 (1983), 473-478.

[3] GRANDE, Z.: Some observations on the symmetrical quasicontinuity of Piotrowski and Vallin, Real Anal. Exchange 31 (2005-2006), 309-314.

[4] GRANDE, Z.: On the continuity of symmetrically cliquish or symmetrically quasicontinuous functions, Real Anal. Exchange 32 (2006-2007), 195-204.

[5] GRANDE, Z:: On the maximal multiplicative family for the class of quasicontinuous functions, Real Anal. Exchange 15 (1989-1990), 437-441.

[6] GRANDE, Z:: On the maximal additive and multiplicative families for the quasicontinuities of Piotrowski and Vallin, Real Anal. Exchange 32 (2007), 511-518.

[7] GRANDE, Z:: On some special notions of approximate continuity, Real Anal. Exchange 24 (1998-1999), 171-183.

[8] GRANDE, Z.-SOLTYSIK, L.: Some remarks on quasicontinuous real functions, Problemy Mat. 10 (1990), 79-86.

[9] IOSIFESCU, M.: Conditions that the product of two derivbatives be a derivative, Rev. Roum. Math. Pures Appl. 4 (1959), 641-649. (In Russian)

[10] KEMPISTY, S.: Sur les fonctions quasicontinues, Fund. Math. 19 (1932), 184-197.

[11] NEUBRUNN, T.: Quasi-continuity, Real Anal. Exchange 14 (1989), 259-306.

[12] PIOTROWSKI, Z.-VALlin, R. W.: Conditions which imply continuity, Real Anal. Exch. 29 (2003-2004), 211-217.

[13] TALL, F. D.: The density topology, Pacific J. Math. 62 (1976), 275-284.

Received November 13, 2008

\author{
Zbigniew Grande \\ The University of Computer Science and \\ Economics TWP Olsztyn \\ ul. Barczewskiego 11 \\ PL-10-061 Olsztyn \\ POLAND \\ E-mail: grande@ukw.edu.pl \\ Ewa Strońska \\ Institute of Mathematics \\ Kazimierz Wielki University \\ Plac Weyssenhoffa 11 \\ PL-85-072 Bydgoszcz \\ POLAND \\ E-mail: stronska@neostrada.pl
}

